\title{
Advanced Colon Cancer after Curative Resection of Intramucosal Adenocarcinoma with Endoscopic Submucosal Dissection
}

\author{
Akiko Sasaki $^{a}$ Chikamasa Ichita $^{a} \quad$ Chihiro Sumida $^{a}$ Karen Kimura $^{a}$ \\ Takashi Nishino $^{a}$ Junichi Tasaki ${ }^{a}$ Sakue Masuda ${ }^{a}$ Jun Kawachi ${ }^{\text {b }}$ \\ Madoka Kudo $^{c}$ Shinichi Teshima ${ }^{c}$ Kazuya Koizumia Makoto Kako ${ }^{a}$ \\ ${ }^{a}$ Gastroenterology Medicine Center, Shonan Kamakura General Hospital, Kamakura, Japan; \\ bDepartment of General Surgery, Shonan Kamakura General Hospital, Kamakura, Japan; \\ 'Department of Diagnostic Pathology, Shonan Kamakura General Hospital, Kamakura, Japan
}

\section{Keywords}

Endoscopic submucosal dissection for colorectal cancer - Interval cancer - Local recurrence .

Postcolonoscopy colorectal cancer · Rapid growth

\section{Abstract}

Endoscopic resection, particularly endoscopic submucosal dissection (ESD), for colorectal cancers enables a precise pathological diagnosis and safe $\mathrm{R} 0$ resection. The recurrence rate after ESD is generally extremely low, with annual surveillance colonoscopy recommended. However, surveillance may not be considered for super-elderly patients owing to their condition. This is a case report of an 85-year-old man in whom curative resection was achieved for an intramucosal adenocarcinoma with ESD. The patient presented with a hypoechoic mass located in his lower right abdomen, diagnosed via surveillance abdominal ultrasound. He had undergone curative ESD for intramucosal cecal cancer 2 years prior. Colonoscopy revealed a type 2 epithelial tumor at the proximal aspect of the ESD scar. lleocolic resection with lymph node dissection was performed. An epithelial tumor and well-differentiated adenocarcinoma but not a submucosal tumor was detected in the mucosal layer. The lesion was diagnosed not as a local recurrence after ESD but as a newly emerged original advanced cancer. After ESD for colorectal cancer, a newly developed advanced cancer may occur at the site of the ESD scar in a shorter term than usual. Surveillance colonoscopy after ESD is necessary even for super-elderly patients. 


\section{Introduction}

Endoscopic submucosal dissection (ESD) for colorectal neoplasms enables a precise pathological diagnosis with en bloc R0 resection. The en bloc resection rate is reported to be $94.5 \%$, and even with an increased lesion size of $>40 \mathrm{~mm}$, it remains over 93\% [1]. After R0 resection by ESD, the recurrence rate is reported to be as low as $2 \%$ after endoscopic piecemeal mucosal resection [2]. Hence, the recurrence rate after ESD for the original colorectal cancer could be extremely low. Radical ESD can be performed for healthy elderly patients over the age of 80 years with colorectal lesions. After ESD for colorectal cancer, annual surveillance colonoscopy is recommended to detect local recurrence and metachronous cancers. However, annual surveillance may not be considered for super-elderly patients, over the age of 80 years, owing to their condition. Herein, we report a case of advanced colon cancer treated with curative resection of the intramucosal adenocarcinoma with ESD.

\section{Case Report/Case Presentation}

An 85-year-old man was referred to our hospital for examination of a hypoechoic mass found in his lower right abdomen during surveillance abdominal ultrasonography. The patient was asymptomatic. He had undergone curative ESD for early cecal cancer 2 years prior. After the ESD procedure, his family physician had performed a surveillance fecal occult blood test and abdominal ultrasound instead of colonoscopy, considering his age. He had a past medical history of hypertension, and his brother was diagnosed with colorectal cancer at an age $>80$ years.

The physical examination and laboratory investigations were unremarkable. The initial lesion resected 2 years prior, via ESD, was a $30 \mathrm{~mm}$, Paris class $0-I I a$, lesion located in the cecum, spanning the ileocecal valve-to-side (Fig. 1). The procedure was performed using a Flush-Knife BT-S $2.0 \mathrm{~mm}$ (Fujifilm Co., Tokyo, Japan). The lesion margins were delineated before ESD using $0.4 \%$ indigo-carmine dye spray and a high-definition, magnified, view with narrow-band imaging. The entire submucosal dissection took $300 \mathrm{~min}$, without the occurrence of adverse events.

The histopathologic diagnosis was adenocarcinoma with tubular adenoma, 0-IIa, $34 \times 24$ $\mathrm{mm}$, tub1, pTis (M), ly0, v0, pHM0, and pVM0. Near the main tumor, curative resection of a 4 $\mathrm{mm}$, Paris class 0 -Is, lesion was also performed in the same surgical specimen, which was diagnosed as a low-grade tubular adenoma measuring $4 \times 3 \mathrm{~mm}$. Colonoscopy performed at the time of resection revealed a type 2-like epithelial tumor at the proximal aspect of the ESD scar (Fig. 2). Biopsy specimens were taken from the lesion and histopathology revealed a well-differentiated adenocarcinoma. Although the lesion was located on the ESD scar, it was an epithelial tumor and not a submucosal tumor. It was diagnosed not as a local recurrence following ESD but as a newly emerged original advanced cancer. Ileocolic resection with lymph node dissection was performed, and the histopathologic diagnosis was adenocarcinoma, type 2, $30 \times 25 \mathrm{~mm}$, muc > tub1 > tub2, pT3 (SS), med, INFa, ly1, v0, pPM0 (20 mm), pDM0 (105 mm), R0, and pN1 (2/20) (Fig. 3). Although the lesion consisted mostly of mucinous carcinoma deeper than the submucosa, a well-differentiated adenocarcinoma was detected in the mucosal layer. Both lesions resected by ESD and surgery were positive for the $K R A S$ codon 12 G12D mutation, based on the RAS mutation analysis. Hence, it was impossible to conclude with the gene analysis that the later advanced cancer had no relationship to the initial early cancer. He was discharged 9 days after surgery without any adverse event. However, after 24 months of follow-up, he suffered a peritoneal dissemination and received palliative therapy.

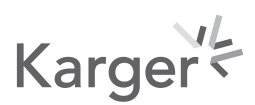



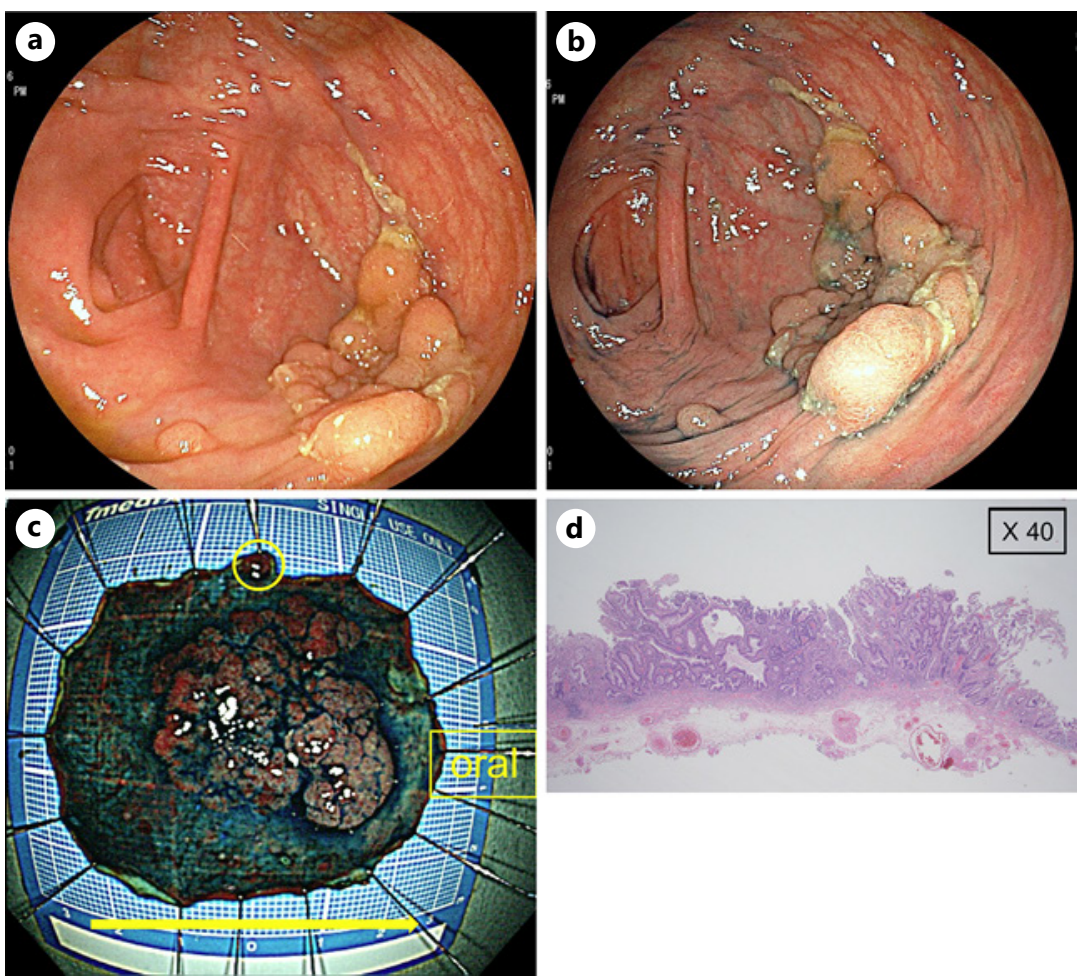

Fig. 1. Initial lesion resected via ESD 2 years prior. a The initial lesion resected 2 years prior via ESD was a $30 \mathrm{~mm}$, Paris class 0-IIa lesion located in the cecum near the ileocal valve near the ileocal valve. $\mathbf{b}$ The lesion margins were delineated using $0.4 \%$ indigo-carmine dye spray, and a $4 \mathrm{~mm} 0$-Is lesion was also detected near the 0 -IIa lesion. c Dissected specimen, $50 \times 40 \mathrm{~mm}$ in diameter, with both of the contained lesions having negative horizontal margins. The proximal aspect is indicated using the right direction arrow. $\mathbf{d}$ A lowpower field image of the resected specimen of the initial lesion resected by ESD is shown. The histopathologic diagnosis was adenocarcinoma with tubular adenoma, 0-IIa, $34 \times 24$ mm, tub1, pTis (M), ly0, v0, pHM0, and pVM0 at a low magnification $(\times 40)$. ESD, endoscopic submucosal dissection.

\section{Discussion/Conclusion}

This case describes the clinical presentation of a patient with a newly emerged advanced cecal cancer on the scar of curative ESD performed 2 years prior for early cecal cancer. It was diagnosed not as a local recurrence but as an original cancer, based on the appearance and histopathologic analysis. The reason for the diagnosis of a newly emerged original cancer on the scar of ESD and surgical anastomosis was based on the observation of a local recurrence and a microscopic lesion near the original tumor.

Local recurrence after ESD for colorectal tumors is reported to be mainly due to residual tumors from the original lesion [3], and rare cases are reported to be caused by the implantation of tumor cells after ESD [4]. Recurrence from the residual tumor could arise from the horizontal margin, lymphatic invasion, and venous invasion [5]. Particularly, lymphatic and vascular invasion are sometimes detected from additional immunostaining or deeper sectioning of the lesion. Regarding the implantation of initial cancer, cells could exfoliate into the intestinal lumen during ESD when the submucosal membrane is pressed close to the tumor cells with a hood to provide traction and a good view for dissection [4]. During colorectal surgery, intraluminal lavage has been reported to be useful for the prevention of implantation at the anastomotic site of rectal cancer. Intraluminal lavage after ESD might also be useful in the prevention of implantation and local 

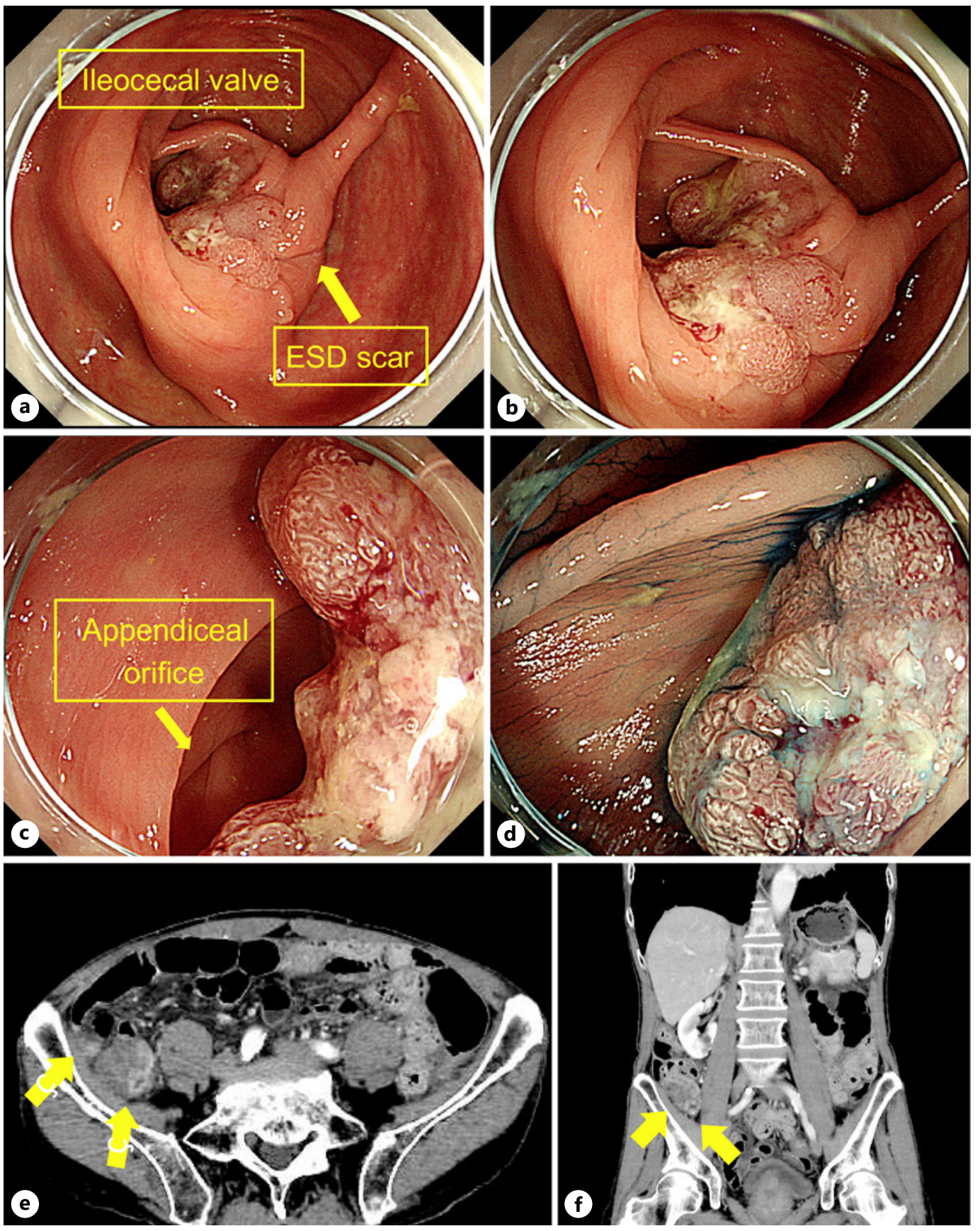

Fig. 2. A follow-up colonoscopy after ESD revealed advanced cecal cancer. a At the 2-year follow-up, a type 2-like epithelial tumor was observed at the proximal aspect of the ESD scar. b Although the lesion was located on the ESD scar, it was not a submucosal tumor but an epithelial tumor. $\mathbf{c}$ The lesion was located between the appendiceal orifice and the ESD scar. $\mathbf{d}$ It was an ulcerative epithelial lesion. Enhanced CT scan showing the poorly and heterogeneously contrasted mass in axial ligation (arrows) (e) and coronal ligation (arrows) (f).

recurrence after ESD [6]. Based on the aforementioned reasons, local recurrence after endoscopic resection usually appears as a submucosal tumor [7]. Histopathologic findings often show the presence of a main lesion in the submucosal layer, while small or no epithelial lesion exists. 

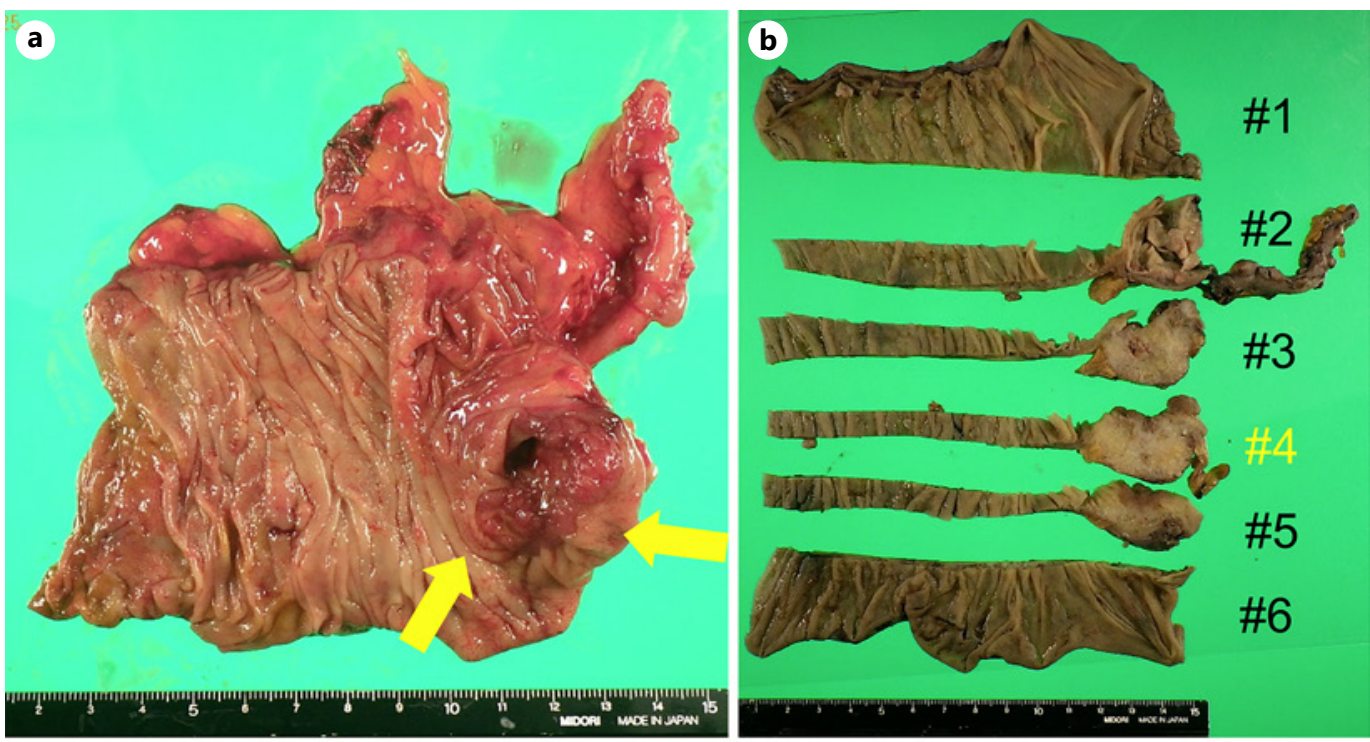

C
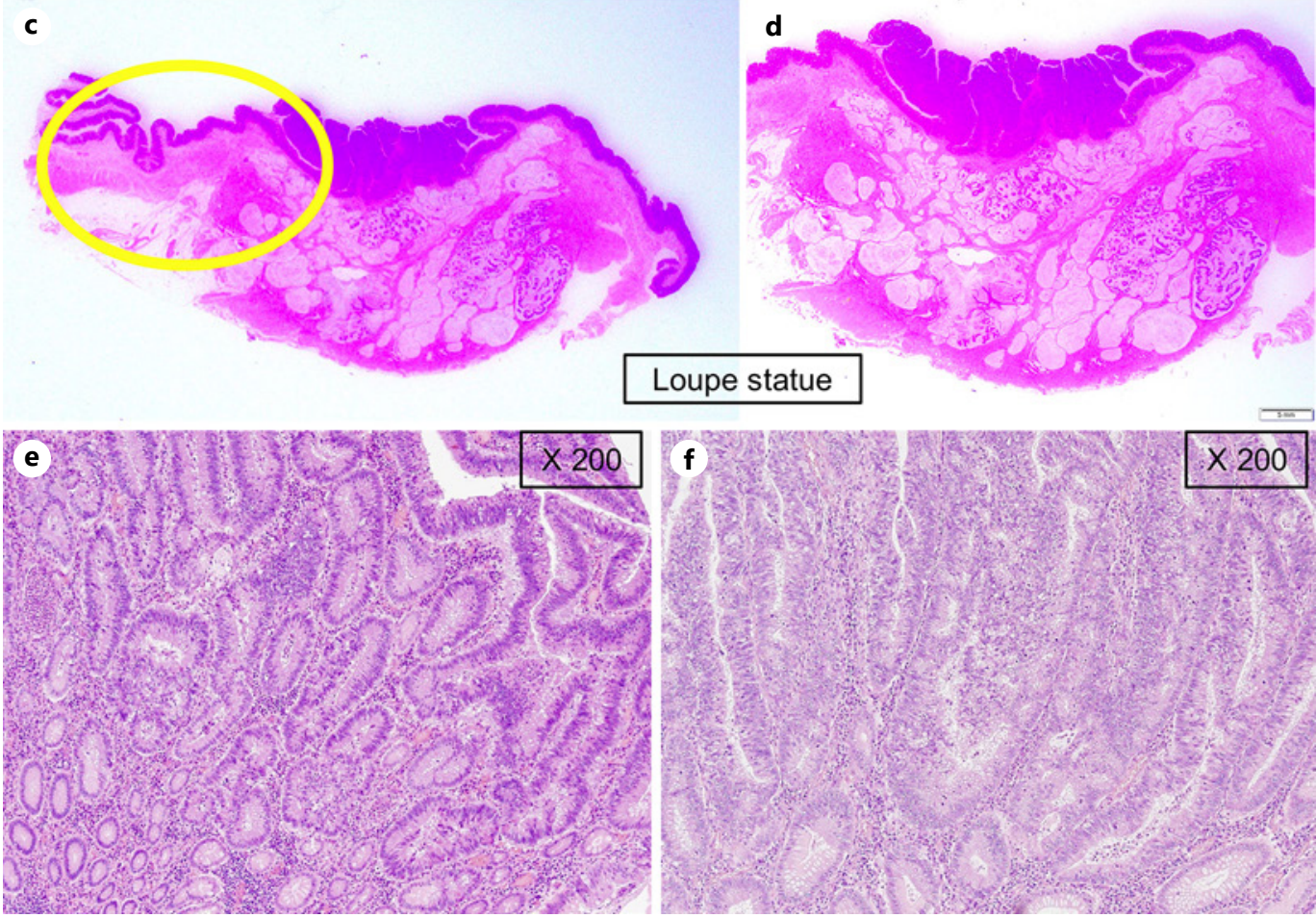

Loupe statue

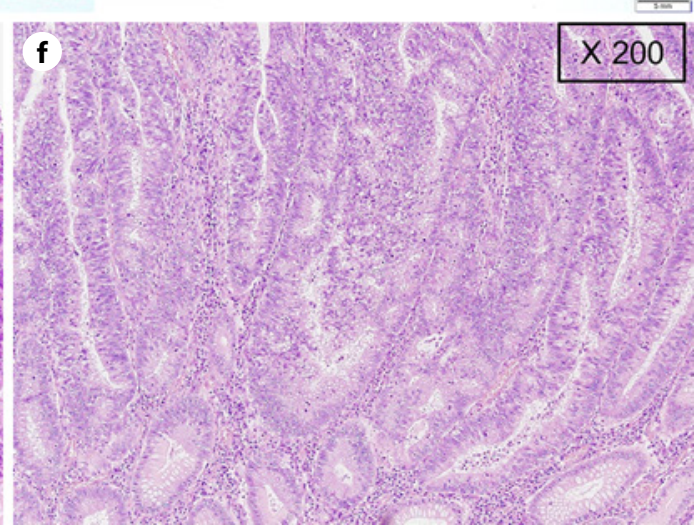

Fig. 3. Histologic and immunohistochemical analyses of a sample from the surgical specimen. a An ileocecectomy specimen including an ulcerative type 2-like lesion located on the ESD scar near the ileocecal valve. $\mathbf{b}$ The \#4 sliced specimen, shown in (c, d). c Loupe image of the resected specimen, with the yellow circle showing the ESD scar. $\mathbf{d}$ The lesion consisted mostly of mucinous carcinoma deeper than the submucosa; a well-differentiated adenocarcinoma was detected in the mucosal layer. e A high-power field image $(\times 200)$ of the surgical specimen is shown. $f$ A high-power field image $(\times 200)$ of the resected specimen of the initial lesion resected by ESD is shown. Both lesions resected by ESD and surgery were positive for the KRAS codon 12 G12D mutation based on RAS mutation analysis. 
In the present case, the lesion at the post-ESD scar was diagnosed as a newly developed cancer because of its endoscopic and histopathologic findings. Interval cancer or postcolonoscopy colorectal cancer and biological reasons may have led to the development of a new cancer. Interval cancer is defined as cancer that develops within 6-60 months following a colonoscopy and is associated with right side of the colon, earlier stage cancer, lower risk of death, higher rate of adenoma, and a family history of CRC [8]. It is related to human error and rapidly growing colorectal cancer, missed lesions, and inadequate preparations, while $13.6 \%$ of the cases have been reported to be newly developed cancers. There are 2 main types of rapidly growing colorectal cancers: serrated lesions, such as sessile serrated adenoma/ polyps which are positive for $\mathrm{CpG}$ island methylator phenotype and have high microsatellite instability [9], and laterally spreading tumors (NG type) in the proximal colon. In our case, a serrated component was not detected histopathologically in the advanced cancer; hence, $\mathrm{CpG}$ island methylator phenotype and microsatellite instability were not examined. It is only presumed that this is a case of a newly developed lesion coincidentally located at the site of an ESD scar. Although RAS mutation analysis did not confirm the difference between the initial and newly developed lesions, any other lesion, including small laterally spreading tumors (NG type), could be detected around the initial lesion apart from the adenoma which was co-resected with the main cancer by ESD.

The present case did not match the typical presentation of interval cancers or postcolonoscopy colorectal cancers. It is unusual that this advanced cancer occurred in only 2 years after ESD. Regarding early gastric cancers, local recurrence with the appearance of original epithelial tumor after curative ESD is reported to be associated with an adjacent neoplastic precursor lesion, the so-called dysplasia-like atypia, but not cases of colorectal cancers [10]. We could not identify dysplasia-like atypia in the initial ESD specimen in this case; however, it is possible that colorectal pit dysplasia adjacent to the ESD scar accelerated the advanced cancer in the course of the ulcer healing process after ESD.

After ESD for colorectal cancer, a newly advanced cancer may develop on the site of the ESD scar over a shorter term than usual. ESD will change the environment around the resected lesion and cause not only local recurrence but also advanced cancer accelerated, rarely, from unrecognized pit dysplasia. Thus, surveillance colonoscopy is necessary after ESD, even if the patient is super-elderly.

\section{Statement of Ethics}

The study was approved by the Shonan Kamakura General Hospital Institutional Review Board, approval number (SKEC-21-05) on April 3, 2021. It was conducted ethically in accordance with the World Medical Association Declaration of Helsinki. Informed consent - written informed consent was obtained from the patient for publication of this case report and any accompanying images.

\section{Conflict of Interest Statement}

The authors have no conflicts of interest to declare.

\section{Funding Sources}

This manuscript did not receive any funding.

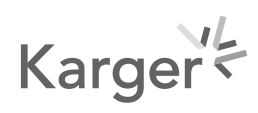




\section{Author Contributions}

Akiko Sasaki, Chikamasa Ichita, Chihiro Sumida, Karen Kimura, Takashi Nishino, Junichi Tasaki, Sakue Masuda, Jun Kawachi, Madoka Kudo, Shinichi Teshima, Kazuya Koizumi, and Makoto Kako made substantial contributions to conception and design, acquisition of data, or analysis and interpretation of data; drafting the article or revising it critically for important intellectual content; and final approval of the version to be published.

\section{References}

1 Nakajima T, Saito Y, Tanaka S, Iishi H, Kudo SE, Ikematsu H, et al. Current status of endoscopic resection strategy for large, early colorectal neoplasia in Japan. Surg Endosc. 2013 Sep;27(9):3262-70.

2 Saito Y, Fukuzawa M, Matsuda T, Fukunaga S, Sakamoto T, Uraoka T, et al. Clinical outcome of endoscopic submucosal dissection versus endoscopic mucosal resection of large colorectal tumors as determined by curative resection. Surg Endosc. 2010 Feb;24(4):343-52.

3 Takao T, Fujiii H, Koyama F, Nakagawa T, Uchimoto K, Nakamura S, et al. Local recurrence of intramucosal cancer in the rectum after en bloc complete resection, report of a case. Clin J Gastroenterol. 2014 Dec;48(13): $36-40$.

4 Oka S, Tanaka S, Kaneko I, Mouri R, Hirata M, Kanao H, et al. Endoscopic submucosal dissection for residual/ local recurrence of early gastric cancer after endoscopic mucosal resection. Endoscopy. 2006 Oct;38(10): 996-1000.

5 Inoue T, Fujii H, Koyama F, Nakagawa T, Uchimoto K, Nakamura S, et al. Local recurrence after rectal endoscopic submucosal dissection: a case of tumor cell implantation. Clin J Gastroenterol. 2014 Dec;7(1):36-40.

6 Inoue T, Fujii H, Koyama F, Nakamura S, Ueda T, Nishigori N, et al. Intraluminal lavage to remove exfoliated tumor cells after colorectal endoscopic submucosal dissection. Surg Endosc. 2016 Jul;30(7):2773-8.

7 Masuda T, Inatsugi N, Yoshikawa S, Uchida H, Kuge H, Yokotani T, et al. A case of advanced transverse colon cancer $7 \mathrm{~mm}$ in diameter resembling a submucosal tumor. Gastroenterol Endosc. 2011;53(9):3047-53.

8 Samadder NJ, Curtin K, Tuohy TM, Pappas L, Boucher K, Provenzale D, et al. Characteristics of missed or interval colorectal cancer and patient survival: a population-based study. Gastroenterology. 2014 Apr;146(4): 950-60.

9 Nishihara R, Wu K, Lochhead P, Morikawa T, Liao X, Qian ZR, et al. Long-term colorectal-cancer incidence and mortality after lower endoscopy. N Engl J Med. 2013 Sep;369:1095-105.

10 Kikuchi S, Kagawa S, Ohara T, Kubota T, Kuwada K, Kagawa T, et al. Recurrence after endoscopic curative resection of mucosal gastric cancer associated with an adjacent neoplastic precursor lesion. Acta Med Okayama. 2016 Jun;70(3):213-6. 